\title{
University of the Witwatersrand physiotherapy undergraduate curriculum alignment to medical conditions of patients within Gauteng state health facilities
}

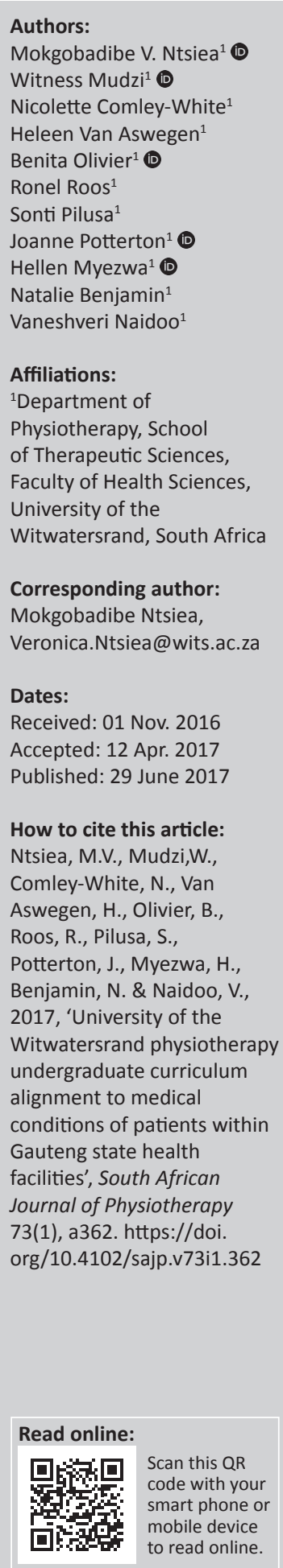

Authors:

Mokgobadibe V. Ntsiea ${ }^{1}$

Heleen Van Aswegen ${ }^{1}$

Benita Olivier ${ }^{1}$ (D)

Natalie Benjamin ${ }^{1}$

Shveri Naidoo ${ }^{1}$

Faculty of Health Sciences,

University of the

Corresponding author:

Mokgobadibe Ntsiea,

Dates:

Accepted: 12 Apr. 2017

How to cite this article:

Ntsiea, M.V., Mudzi,W.,

Comley-White, N., Van

Aswegen, H., Olivier, B.,

Roos, R., Pilusa, S.

Potterton, J., Myezwa, $H_{\text {, }}$

Benjamin, N. \& Naidoo,

Witwatersrand physiotherapy

undergraduate curriculum

alignment to medical

Gauteng state health

facilities', South African

Journal of Physiotherapy

73(1), a362. https://doi.

org/10.4102/sajp.v73i1.362

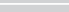

to read online.
Background: The healthcare sector requires graduates with the ability to confidently assess and manage the majority of the medical conditions seen in hospitals.

Objective: To establish whether the most prevalent medical conditions treated by physiotherapists in Gauteng (South Africa) state health facilities align with the University of the Witwatersrand (Wits) physiotherapy curriculum.

Methods: This was a retrospective review of condition-related statistics from physiotherapy departments within the Gauteng province state health facilities. Data from all Gauteng government hospitals that had submitted at least $75 \%$ of their physiotherapy condition-related statistics to the provincial statistics coordinator from January 2012 to December 2014 were considered and compared to medical conditions covered in the Wits 2015 physiotherapy curriculum to check if all conditions listed in the Gauteng statistics appeared within the Wits curriculum document. The number of teaching hours for the common conditions was noted to check the emphasis given to these conditions in the curriculum.

Results: Eighty-three per cent of the hospitals submitted $75 \%$ of their monthly statistics. Overall, the most common conditions treated were lower limb fractures (13\%) followed by stroke $(7.6 \%)(n=705$ 597). Within the neuro-musculoskeletal category, the most common conditions after lower limb fractures were soft tissue injuries (15.1\%) ( $n=330511)$. The most common cardiopulmonary conditions were tuberculosis $(24.9 \%)$, followed by pneumonia $(13.8 \%)(n=94895)$. The most common neurological conditions were stroke $(30.9 \%)$ followed by cerebral palsy $(17 \%)(n=174024)$. Within the non-specified categories, the number of intensive care unit (ICU) patients was the highest (23\%), followed by sputum induction (21\%) ( $n=138$ 187). The most common conditions that were emphasised within the Wits curriculum as indicated by the teaching hours: fractures, 14.5 (66\%) of 22 third-year orthopaedics hours; stroke, 30 (73\%) of 41 third-year neurology hours; soft tissue injuries, 18 (38\%) of 48 fourthyear neuro-musculoskeletal hours; back lesions, 24 (50\%) of 48 fourth-year neuromusculoskeletal hours; and ICU patients, 30 (79\%) of 38 fourth-year cardiopulmonary hours.

Conclusion: The Wits physiotherapy curriculum covers all medical conditions treated by physiotherapists within the Gauteng state health facilities, and overall, the curriculum prepares the students to practise in a variety of situations.

\section{Introduction}

The development of physiotherapy is dependent on the abilities and potential of the graduates, and these graduates need to have skills that are relevant to the needs of the health sector to enable them to practise competently and with confidence (Hunt et al. 1998). Many physiotherapy patients present with cardiopulmonary, neurological and musculoskeletal conditions, and thus academic programmes need curricula that facilitate student learning in preparation for these conditions; however, specific conditions to be covered should be appropriate to the current and future needs of their country's health sector (Council of Canadian Physiotherapy Programmes 2009). Therefore, if our graduates cannot manage conditions seen in the South African (SA) health sector, they will not be relevant.

The healthcare sector requires physiotherapy graduates who can enter its employ with the ability to confidently assess and manage the majority of medical conditions seen in its hospitals.

Copyright: @ 2017. The Authors. Licensee: AOSIS. This work is licensed under the Creative Commons Attribution License. 
Universities aim to equip graduates with broad generic, transferable skills in preparation for a path of lifelong learning (Cowan, Norman \& Coopmah 2005). Undergraduate training, especially curriculum content is thus instrumental in order to meet the needs of both the employer while ensuring lifelong learning for the graduates (Higgs, Higgs \& Neubauer 1999). Therefore, to meet the needs of the healthcare sector, a curriculum is supposed to have current clinical skills as well as cover a wide variety of medical conditions that will be encountered during clinical practice (Brown, Brown \& Roever 2006). Thus, review of the curriculum to meet the national needs is required for relevance of training of the health professionals (Mumbo \& Kinaro 2015). Failure of the curriculum to keep pace with the national health conditions leads to the deterioration of the quality of graduates (Awases et al. 2013). However, in keeping pace with the national needs, all conditions and clinical skills must be fitted within the 480 credits of training over the four years of study.

As the content of the undergraduate curriculum expands to accommodate the changes in disease burden and the growth in knowledge, it should be remembered that the length of the course remains the same and thus the programme should not be overloaded (Chipchase, Williams \& Robertson 2008). When adding additional medical conditions to the curriculum, it is important to consider evidence that supports management of these conditions. However, it has to be noted that there is no clear and accepted model for curriculum decision-making based on best available research evidence (Chipchase et al. 2008). Thus, in some instances, if there is evidence of benefit to the patient, albeit low-level evidence, some medical conditions may be included in the curriculum if they are common because training should meet the needs of local communities within their unique context (Pinnock \& Jones 2008).

When developing or revising a curriculum, a good starting point would be the determination of the diagnoses or medical conditions that are appropriate to include or exclude in the undergraduate physiotherapy degree within a SA context in order to be responsive to the national disease burden. This is needed so that the universities can produce graduates who are well prepared for clinical work within SA while also being prepared to cope in a variety of clinical contexts outside of SA. This is supported by the Witwatersrand (Wits) physiotherapy department mission statement, which refers to graduating physiotherapists committed to meeting the health needs of all communities in SA, appropriately and cost effectively. Significant sociopolitical changes in the 1990s in SA prompted the Wits department of physiotherapy to begin the process of questioning the relevance and appropriateness of its undergraduate curriculum (Stewart et al. 1994; Wallner \& Stewart 1994). This interrogation has been ongoing.

Each physiotherapy department within the Gauteng state hospitals is expected to submit monthly patient conditionspecific statistics to a central provincial coordinator. Information gained from the patient statistics is used for health system monitoring and planning purposes such as motivation for posts and determining in-service training requirements (Health Metrics Network 2008). Physiotherapy training institutions can also use this information to check the relevance of the training programmes in addressing the most common medical conditions or injuries within our communities. Thus, information gained from the patient statistics can be used to inform curriculum content for training institutions. This information can also be used to check if the scope of the physiotherapy minimum training standard as set by the Health Professions Council of SA covers most of the medical conditions within the SA government hospitals. Ideally, data to make such decisions should be based on national patient statistics. However, each province has its own physiotherapy data capturing system, which differs from province to province, and thus it is difficult to make comparisons across provinces.

Relevant data for patient statistics may come from the national-level database of patients' medical conditions that are captured through the SA District Health Information System (DHIS). However, most of the DHIS data are not physiotherapy specific. The DHIS does not capture neuromusculoskeletal, neurological or orthopaedic conditions. In some instances, data that relate to physiotherapy are captured as mortality rates instead of morbidity such as in the study by Bradshaw et al. (2010) where the top six causes of death in SA are reported as HIV/AIDS, tuberculosis, pneumonia, diabetes, stroke and obstructive lung diseases. For the purposes of this study, DHIS data were not used because we needed morbidity data for all clinical areas of physiotherapy (musculoskeletal, neurological and orthopaedics).

Only data from Gauteng hospitals were considered in this study. Primarily because physiotherapy patient statistics are not collated nationally, and thus there is no standardised data collection sheet for all provinces. So, data from the Gauteng province were analysed as a starting point until such time that there is a standardised physiotherapy data collection sheet across provinces. Gauteng is the province of choice for this study as it has the largest SA population (South African Statistics 2012).

The aim of this study was to establish whether the most prevalent medical conditions treated by physiotherapists in Gauteng state health facilities were covered in the Wits physiotherapy curriculum.

\section{Methods}

This was a retrospective review based on condition-related statistics collected from physiotherapy departments within the Gauteng province state health facilities. To review the Wits curriculum, we looked at the content of the curriculum to identify medical conditions covered for each clinical area. Data from all Gauteng government hospitals that submitted at least $75 \%$ (i.e. at least nine months per year) of their physiotherapy condition-related statistics to the provincial 
statistics coordinator from January 2012 to December 2014 were considered and compared to medical conditions covered within the Wits 2015 physiotherapy curriculum. Teaching timetables were also checked in case there were medical conditions that appeared on timetables that were not in the curriculum or course objectives. The amount of teaching time allocated to the most common conditions was noted from the physiotherapy teaching timetables.

A condition-related data capturing sheet was used to capture data for conditions treated by physiotherapists within the Gauteng government health facilities. The total number of patients seen during this time (2012-2014) was noted and the names of the health facilities were captured separately to ensure anonymity. Wits summary of the medical conditions was captured and organised into categories similar to those for the Gauteng health facility statistics (neuromusculoskeletal, cardiopulmonary, neurology, including paediatrics for each category, other or non-specified categories).

A research assistant approached the chief physiotherapist who is responsible for collating physiotherapy statistics for Gauteng to acquire the required data. Three data collection researchers scanned the data for relevant information and captured the information on a spreadsheet, which was used to interpret results. Data from the hospitals were summarised using frequencies and percentages and university data were checked to establish if the most common hospital conditions appeared within the curriculum. The amount of time allocated for teaching the most common conditions was noted to check the emphasis of these conditions within the curriculum.

\section{Results}

Data were received from the following Gauteng municipalities: City of Johannesburg metropolitan, City of Tshwane metropolitan, Ekurhuleni metropolitan, Sedibeng district, Emfuleni local, Lesedi local, Midvaal local, Metsweding district, Kungwini, Nokeng tsa Taemane, West Rand district, Merafong city local, Mogale city local, Randfontein local and Westonaria local.

Data were expected from 30 facilities. In 2012, 28 facilities (93\%) submitted but only 21 (75\%) of these facilities submitted more than $75 \%$ of their statistics (i.e. minimum of 9 months out of the expected 12 months). In 2013, 28 facilities (93\%) submitted, but only 15 (54\%) had more than 75\%. In 2014, only April-December statistics were available for the researchers, and 25 hospitals (83\%) submitted, with 17 (68\%) above $75 \%$ of their expected submissions. Overall, 25 facilities' data were used.

Of the total conditions, the most common conditions treated by physiotherapists within the Gauteng province state health facilities were lower limb fractures (including ankles) (13\%) followed by stroke (7.6\%) and soft tissue injuries (7.1\%) (Table 1). The most common neuromusculoskeletal conditions were lower limb fractures (27.7\%) followed by soft tissue injuries (15.1\%) and upper limb fractures $(12.9 \%)$ (Table 2). The least common neuromusculoskeletal conditions were facial fractures $(0.2 \%)$, brachial plexus lesions $(0.5 \%)$ and traumatic amputations $(0.5)$.

The most common cardiopulmonary conditions were tuberculosis $(24.9 \%)$, followed by pneumonia $(13.8 \%)$ and patients with intercostal drains (ICDs) (13.8\%) (Table 3). The least common cardiopulmonary conditions were organ transplants $(0.1 \%)$ and laryngectomy $(0.1 \%)$.

The most common neurological conditions were stroke (30.9\%) followed by cerebral palsy (17\%) and developmental delay $(11.8 \%)$ (Table 4$)$. The least common conditions were general central nerve lesions (excluding spinal cord injury, head injury and stroke) (2.7\%) and Bell's palsy $(0.7 \%)$.

Within the non-specified categories, the number of intensive care unit (ICU) patients was the highest (23\%), followed by sputum induction (21\%) and general weakness (10.6) (Table 5).

TABLE 1: The most common conditions treated by physiotherapists in the Gauteng state hospitals during the period 2012-2014 ( $n=705597)$.

\begin{tabular}{|c|c|c|}
\hline Medical conditions & $n$ & $\%$ \\
\hline Lower limb fractures (including ankles) & 91705 & 13.0 \\
\hline Stroke & 53794 & 7.6 \\
\hline Soft tissue injuries & 49894 & 7.1 \\
\hline Upper limb fractures (excluding hands) & 42765 & 6.1 \\
\hline Arthritic conditions: osteoarthritis & 35156 & 5.0 \\
\hline Back (discogenic lesions) & 34192 & 4.8 \\
\hline Intensive care unit patients & 32020 & 4.5 \\
\hline Cerebral palsy & 29692 & 4.2 \\
\hline Sputum induction or collection & 29273 & 4.1 \\
\hline Tuberculosis & 23598 & 3.3 \\
\hline Developmental delay & 20516 & 2.9 \\
\hline Hand injuries & 20343 & 2.9 \\
\hline Head injuries & 18727 & 2.7 \\
\hline General weakness & 14669 & 2.1 \\
\hline Burns & 14233 & 2.0 \\
\hline Neurosurgery & 14217 & 2.0 \\
\hline Pneumonia & 13062 & 1.9 \\
\hline Intercostal drains & 13061 & 1.9 \\
\hline Mastectomy & 12315 & 1.7 \\
\hline Amputations: vascular & 11923 & 1.7 \\
\hline Spinal lesions (non-traumatic) & 10309 & 1.5 \\
\hline Acute lung disease & 8764 & 1.2 \\
\hline Joint replacements & 8003 & 1.1 \\
\hline Peripheral nerve lesions & 7524 & 1.1 \\
\hline Spinal injuries (traumatic) & 7180 & 1.0 \\
\hline Routine pre- or -post-laparatomy & 6827 & 1.0 \\
\hline Othert & 81835 & 11.6 \\
\hline Total & 705597 & 100.0 \\
\hline
\end{tabular}

Only conditions $>1 \%$ are presented in this table.

$\dagger$, Other: Neonates, Meningitis, High Care or Ortho Acute, Central Nerve Lesions, Cardiothoracic Surgery or Trauma, Discogenic Lesions, Pelvic fractures, Dislocations, Vertebral fractures (no fall-out), Congenital disorders, Lung Abscess, Rheumatoid arthritis, Caesarean + fractures (no fall-out), Congenital disorders, Lung Abscess, Rheumatoid arthritis, Caesarean +
Gynae, Chronic obstructive pulmonary disease, Spinal surgery, Pre- or -post-natal, Circulatory disorders, Soft tissue reconstructive surgery, Bronchiectasis, Cardiac rehab, Peripheral nerve injuries, Oncology, Asthma, Chronic pain, Chronic lung disease, Extensive general surgery, Amputations: traumatic, Incontinence, Cystic fibrosis, Nephrology, Bell's palsy, Wound care, Dermatology, Sinusitis, Haemophilia, Urology, Visual impairment, Facial fractures, Stress, Obesity, Laryngectomy, Malaria, Organ transplants. 
TABLE 2: Neuro-musculoskeletal conditions (including paediatric cases) $(n=330511)$.

\begin{tabular}{lcc}
\hline Medical conditions & $\boldsymbol{n}$ & $\mathbf{\%}$ \\
\hline Lower limb fractures (including ankles) & 91705 & 27.7 \\
Soft tissue injuries & 49894 & 15.1 \\
Upper limb fractures (excluding hands) & 42765 & 12.9 \\
Arthritic conditions: Osteoarthritis & 35156 & 10.6 \\
Back (discogenic lesions) & 34192 & 10.3 \\
Hand injuries & 20343 & 6.2 \\
Amputations: vascular & 11923 & 3.6 \\
Joint replacements & 8003 & 2.4 \\
Neck (discogenic lesions) & 4677 & 1.4 \\
Pelvic fractures & 4202 & 1.3 \\
Dislocations & 4145 & 1.3 \\
Vertebral fractures (no neurological fall-out) & 3891 & 1.2 \\
Congenital disorders & 3770 & 1.1 \\
Arthritic conditions: Rheumatoid arthritis & 3740 & 1.1 \\
Spinal surgery (including laminectomy) & 2977 & 0.9 \\
Soft tissue reconstructive surgery & 2691 & 0.8 \\
Peripheral nerve injuries (excluding brachial plexus) & 2394 & 0.7 \\
Amputations: traumatic & 1742 & 0.5 \\
Brachial plexus & 1724 & 0.5 \\
Facial fractures (temporomandibular joint/dislocations) & 577 & 0.2 \\
\hline Total & 330511 & 100.0 \\
\hline
\end{tabular}

TABLE 3: Cardiopulmonary conditions (including paediatric cases) $(n=94895)$.

\begin{tabular}{lcc}
\hline Medical conditions & $\boldsymbol{n}$ & $\mathbf{\%}$ \\
\hline Tuberculosis & 23598 & 24.9 \\
Pneumonia & 13062 & 13.8 \\
Intercostal drains & 13061 & 13.8 \\
Acute lung disease & 8764 & 9.2 \\
Routine pre- or -post-laparatomy & 6827 & 7.2 \\
Cardio-thoracic surgery or trauma & 4694 & 4.9 \\
Lung abscess & 3762 & 4.0 \\
Chronic obstructive pulmonary disease & 3300 & 3.5 \\
Bronchiectasis & 2529 & 2.7 \\
Myocardial infarction and cardiac rehab & 2477 & 2.6 \\
Asthma & 2365 & 2.5 \\
Chronic lung disease & 1962 & 2.1 \\
Extensive general surgery & 1852 & 2.0 \\
Cystic fibrosis & 1665 & 1.8 \\
Head and neck conditions that affect respiration & 1408 & 1.5 \\
Vascular conditions that affect respiration & 1186 & 1.2 \\
Sinusitis & 1179 & 1.2 \\
Urology & 982 & 1.0 \\
Laryngectomy & 124 & 0.1 \\
Organ transplants & 98 & 0.1 \\
\hline Total & 94895 & 100.0 \\
\hline
\end{tabular}

The least common conditions were malaria $(0.1 \%)$, obesity $(0.1 \%)$ and stress $(0.3 \%)$.

Almost all the conditions listed on the Gauteng physiotherapy patients' condition statistics are included within the Wits curriculum summary (Appendix 1). Only patients whose primary condition was stress and laryngectomy are not explicitly covered within the Wits curriculum. The most common conditions were emphasised within the Wits curriculum as indicated by the amount of teaching time allocated for these common conditions: fractures, $14.5(66 \%)$ of 22 third-year orthopaedics hours; stroke, 30 (73\%) of 41 third-year neurology hours; soft tissue injuries, 18 (38\%) of
TABLE 4: Neurological conditions (including paediatric cases) $(n=174024)$.

\begin{tabular}{lcc}
\hline Medical conditions & $\boldsymbol{n}$ & $\mathbf{\%}$ \\
\hline Stroke & 53794 & 30.9 \\
Cerebral palsy & 29692 & 17.1 \\
Developmental delay & 20516 & 11.8 \\
Head injuries & 18727 & 10.8 \\
Neurosurgery & 14217 & 8.2 \\
Spinal lesions (non-traumatic) & 10309 & 5.9 \\
Peripheral nerve lesions & 7524 & 4.3 \\
Spinal injuries (traumatic) & 7180 & 4.1 \\
Meningitis & 6069 & 3.5 \\
Central nerve lesions & 4711 & 2.7 \\
Bell's palsy & 1285 & 0.7 \\
\hline Total & $\mathbf{1 7 4 0 2 4}$ & $\mathbf{1 0 0 . 0}$
\end{tabular}

TABLE 5: Conditions from non-specified categories (including paediatric cases) ( $n=138$ 187).

\begin{tabular}{lcc}
\hline Medical conditions & $\boldsymbol{n}$ & $\boldsymbol{\%}$ \\
\hline Intensive care unit patients & 32020 & 23.2 \\
Sputum induction or collection & 29273 & 21.2 \\
General weakness & 14669 & 10.6 \\
Burns & 14233 & 10.3 \\
Mastectomy & 12315 & 8.9 \\
Early intervention or neonates & 6128 & 4.4 \\
High care and acute ortho & 4956 & 3.6 \\
Caesarean + Gynae & 3387 & 2.5 \\
Pre- or -post-natal & 2906 & 2.1 \\
Circulatory disorders (including hypertension) & 2705 & 2.0 \\
Oncology & 2382 & 1.7 \\
Chronic pain & 2240 & 1.6 \\
Diabetes & 1813 & 1.3 \\
Incontinence & 1683 & 1.2 \\
Nephrology (dialysis) & 1578 & 1.1 \\
\hline Wound care & 1280 & 0.9 \\
Dermatology & 1241 & 0.9 \\
\hline Haemophilia & 1077 & 0.8 \\
\hline Other (non-specified conditions) & 906 & 0.7 \\
Visual impairment & 744 & 0.5 \\
Stress & 386 & 0.3 \\
Obesity & 148 & 0.1 \\
\hline Malaria & 117 & 0.1 \\
\hline Total & & 100.0 \\
\hline & & \\
\hline
\end{tabular}

48 fourth-year neuro-musculoskeletal hours; back lesions, $24(50 \%)$ of 48 fourth-year neuro-musculoskeletal hours; and ICU patients, 30 (79\%) of 38 fourth-year cardiopulmonary hours.

\section{Ethical consideration}

Ethical clearance (M140870) was granted by the University of the Witwatersrand Human Research Ethics Committee for research on human subjects and permission to use the physiotherapy patient statistics was granted by the Gauteng health department. The physiotherapists responsible for collation of the physiotherapy patient statistics were contacted to make them aware of the provincial department permission and to request their permission and time to discuss the statistics. The study proposal was presented at the Gauteng Physiotherapy Forum (2014/03/11) to share the plan with Gauteng physiotherapists and to get their advice on possible errors to be aware of when collating the data. 


\section{Discussion}

With this paper, we aimed to establish whether the most prevalent medical conditions treated by physiotherapists in Gauteng state health facilities were covered in the Wits physiotherapy curriculum. Eighty-three per cent (25) of the hospitals submitted more than $75 \%$ of their monthly statistics over the 2012-2014 data collection period. This information can thus be used to reflect on the type of patients seen by physiotherapists within the Gauteng state hospitals because data are from almost all hospitals within the province.

The Wits physiotherapy curriculum covers all medical conditions treated by physiotherapists within the Gauteng state health facilities and also covers everything within the World Confederation of Physical Therapy (WCPT) minimum training standards (WCPT 2011). Emphasis on the most common conditions within the Wits physiotherapy curriculum is on the most common conditions treated by physiotherapists within the Gauteng state health facilities as indicated by the number of physiotherapy teaching hours allocated for these conditions.

Conditions that are least common within these facilities are also included in the Wits curriculum and this prepares the students to practise in all clinical settings within and outside SA. Examples of these conditions are malaria, which is less common in SA compared to other regions within subSaharan Africa (SA Malaria Report 2013). Multiple sclerosis (MS) is also less common in SA but more common in North America and Europe (Atlas of Multiple Sclerosis 2013) and this explains why the number of patients with these conditions is low in the patient statistics for the province. However, these conditions, even though not part of the core curriculum, are covered within the curriculum to prepare students for the global population and the few cases they may encounter within SA. Students can assess patients well and thus if they come across unusual conditions they can cope because they can identify the patient's problems and related impairments.

Medical conditions seen by physiotherapists within the Gauteng state health facilities are similar to the national disease burden and Wits curriculum. Thus, Wits is teaching what is relevant to our health sector and thus contributes graduates who are useful for the country. Tuberculosis and pneumonia are the most common cardiopulmonary conditions in this study. This may be because of the fact that these conditions are HIV-opportunistic diseases (Global AIDS Response Progress Reporting 2015), and with a high number of people living with HIV in SA (UNAIDS South African 2015), it makes sense that these are among the most common conditions treated by physiotherapists. Bradshaw et al. (2010) also included these conditions in their top six causes of death in SA. Stroke is also one of the most common conditions seen by physiotherapists, and this is expected because of the high rate of hypertension, diabetes, obesity and HIV, which are all risk factors for stroke (Connor et al. 2005; Ortiz et al. 2007).
HIV, depression, ischaemic heart diseases and road traffic accidents are the top four 2030 projected causes of burden of disease (Mathers \& Loncar 2006). All these conditions or conditions associated with these (e.g. fractures and soft tissue injuries from road traffic accidents, stroke because of heart diseases of HIV) are covered within the Wits physiotherapy curriculum. This addresses what was raised by Stewart et al. (1994) in their study to determine Wits physiotherapy graduates' contribution to SA's health needs. They concluded that the graduates over-serviced soft tissue, orthopaedics and respiratory conditions; however, our study shows that these are indeed among the most common conditions. Stewart et al. (1994) also indicated that neurological, spinal cord lesions and cerebral palsy were underserviced. In our study, these conditions are also among the most common conditions treated by physiotherapists and are also covered within the Wits curriculum. Thus, Wits graduates do meet the needs of the current SA health sector.

Fractures, soft tissue injuries and patients with ICDs are also common. This is expected because of the high trauma rate in SA (motor vehicle accidents, stab chest and occupational injuries) (Norman et al. 2007). The high number of ICU patients may be because of the fact that almost all patients in ICU require physiotherapy for chest clearance or mobilisation. There was also a high number of sputum inductions, and this may be because of the fact that tuberculosis is the most common cardiopulmonary condition and some of the patients cannot cough productively; yet, their sputum is required for laboratory tests to confirm diagnosis. Wits students are taught sputum induction and thus will be able to collect sputum whenever indicated.

Developmental delay was also common in this study, and this could be in part because of HIV (Potterton et al. 2010). Children in SA are vulnerable to several other factors causing developmental delay including malnutrition and poverty (Walker et al. 2011). Conditions that appear on the Gauteng patient statistics form that are not explicitly covered within the Wits curriculum are stress and laryngectomy. These are covered indirectly because students learn skills to manage symptoms of patients who may present with stress such as pain, muscle spasm, poor endurance and somatoform disorders and also teach patients exercises and personal management to prevent or manage the stress-related impairments or problems (Kaur, Masaunz \& Bhatia 2013). This is covered during the community physiotherapy block; however, after graduating, students have opportunities to do short courses if they have to focus on stress management.

Laryngectomy is not covered, but students do cover respiratory techniques including suctioning that are required for the management of patients post-laryngectomy. Laryngectomy is usually performed for patients with cancer of the larynx, and in oncology, they cover the systemic effects of cancer and the role of physiotherapy in treating these. Patients with laryngectomy may have a tracheostomy in place and tracheostomy management and care is also part of 
the Wits ICU curriculum. The focus in the Wits curriculum is primarily on lung, breast and cervical cancer as these are the most common types of cancers. However, graduates will be able to cope with any cancer condition that requires cardiopulmonary, neurological or musculoskeletal clinical skills because they can assess and identify problems and possible causes.

The results of this study indicate that all conditions treated by physiotherapists within the Gauteng province are covered by the Wits physiotherapy curriculum. The curriculum actually covers more conditions than those that appear on the Gauteng physiotherapy patient statistics form, and this is to ensure that students are trained to work in any clinical facility throughout the world even though the core business is the SA context. Thus, the Wits physiotherapy curriculum accommodates the necessary generic skills to ensure mobility and enhance quality of life.

All medical conditions identified as the most common by Bradshaw et al. (2010) are covered within both the Wits curriculum and Gauteng statistics form. The conditions are also in line with what the WCPT considers within the minimum physiotherapy training standards as indicated in this portion of the WCPT (2011) document:

The physical therapist professional curriculum includes content and learning experiences in the clinical sciences (e.g. content about the cardiovascular, pulmonary, endocrine, metabolic, gastrointestinal, genitourinary, integumentary (skin), musculoskeletal and neuromuscular systems and the medical and surgical conditions frequently seen by physical therapists). (p. 11)

\section{Limitations of the study}

This was a record review, and thus, the authors did not have control over the development, submission and collation of provincial statistics.

\section{Conclusion}

The Wits physiotherapy curriculum covers all medical conditions treated by physiotherapists within the Gauteng state health facilities. Conditions that are least common within these facilities are also included in the Wits curriculum, and this prepares the students to practise in other worldwide clinical settings where such conditions are common. The conditions are also in line with what the WCPT considers to be the minimum physiotherapy training standards. Medical conditions treated by physiotherapists within the Gauteng state health facilities are within the Wits curriculum and on the list of the national and international disease burden.

\section{Acknowledgements}

Ms Heidi van Zyl for summarising data from the Gauteng province, Ms Elma Burger for helping with the process of getting permission from Gauteng Department of Health to use the statistics, Ms Retha Jacobs for availing the data collated from Gauteng hospital physiotherapy departments and Gauteng physiotherapists for capturing and submitting patients' statistics. Your help is much appreciated.

\section{Competing interests}

The authors declare that they have no financial or personal relationships that may have inappropriately influenced them in writing this article.

\section{Authors' contributions}

M.V.N. was responsible for the conception of the study, acquisition of data, drafting of the manuscript. M.V.N., W.M. and N.C. were responsible for analysis of data. All authors, M.V.N., W.M., N.C., H.V., B.O., R.R., S.P., J.P., H.M., N.B. and V.N., approved the design of the study, revision and final approval of the manuscript.

\section{References}

Atlas of Multiple Sclerosis, 2013, Mapping multiple sclerosis around the world, Multiple Sclerosis International Federation, viewed 25 October 2016, from https://www.msif.org/wp-content/uploads/2014/09/Atlas-of-MS.pdf

Awases, M.H., Bezuidenhout, M.C. \& Roos, J.H., 2013, 'Factors affecting the performance of professional nurses in Namibia', Curations 36(1), 108. https://doi. org/10.4102/curationis.v36i1.108

Bradshaw, D., Pillay-Van Wyk, V., Laubscher, R., Nojilana, B., Groenewald, P., Nannan, N., et al., 2010, Cause of death statistics for South Africa: Challenges and possibilities for improvement, Medical Research Council of South Africa: Burden of Disease Research Unit Report, viewed 20 March 2017, from http://www.mrc.ac.za/bod/ cause_death_statsSA.pdf

Brown, G.T., Brown, A. \& Roever, C., 2006, 'A review of paediatric occupational therapy university curricula in South Africa: Part one', International Journal Therapy Rehabilitation 13, 103-108. https://doi.org/10.12968/ijtr.2006.13.3.21361

Chipchase, L.S., Williams, M.T. \& Robertson, V.J., 2008, 'A framework for determining curricular content of entry level physiotherapy programmes: Electrophysical agents as a case study', Physical Therapy Reviews 13, 386-394. https://doi.org/10. $1179 / 174328808 \times 309269$

Connor, M., Rheeder, P., Bryer, A., Meredith, M., Beukes, M., Dubb, A. et al., 2005, 'The South African stroke risk in general practice study', South African Medical Journal 95, 334-339.

Council of Canadian Physiotherapy Programmes, 2009, Entry to practice physiotherapy curriculum: Content guidelines for Canadian University programmes, viewed 24 March 2017, from www.physiotherapyeducation.ca/Resources/National\%20 PT\%20Curriculum\%20Guidelines\%202009.pdf

Cowan, D.T., Norman, I. \& Coopmah, V.P., 2005, 'Competence in nursing practice: A controversial concept -A focused review of the literature', Nurse Education Today 25(5), 355-362. https://doi.org/10.1016/j.nedt.2005.03.002

Global AIDS response progress reporting, 2015, viewed 27 October 2016, from http:// www.unaids.org/sites/default/files/media_asset/JC2702_GARPR2015guidelines_ en.pdf

Health Metrics Network, 2008, Framework and standards for country health information systems, Geneva: World Health Organization, viewed 20 February 2017, from http://apps.who.int/iris/handle/10665/43872

Higgs, J.A.H., Higgs, J. \& Neubauer, D., 1999, 'Physiotherapy education in the changing international healthcare and educational contexts', Advances in Physiotherapy 1 , 17-26.

Hunt, A., Adamson, B., Higgs, J. \& Harris, L., 1998, 'University education and physiotherapy professional', Physiotherapy 84(6), 264-273. https://doi.org/10. 1016/S0031-9406(05)65527-7

Kaur, J., Masaunz, M. \& Bhatia, M.S., 2013, 'Role of physiotherapy in metal health disorders', Delhi Psychiatry Journal 16(2), viewed 24 March 2017, from http:// medind.nic.in/daa/t13/i2/daat13i2p404.pdf

Mathers, C.D. \& Loncar, D., 2006, 'Projections of global mortality and burden of disease from 2002 to 2030', PLoS Medicine 3(11), e442. https://doi.org/10.1371/ journal.pmed.0030442

Mumbo, H.M. \& Kinaro, J.W., 2015, 'Assessment of quality and relevance of curricula development in health training institutions: A case study of Kenya', Human Resources for Health 13, 67. https://doi.org/10.1186/s12960-015-0048-9

Norman, R., Matzopoulos, R., Groenewald, P. \& Bradshaw, D., 2007, 'The high burden of injuries in South Africa', Bulletin World Health Organization 85(9), 695-702. https://doi.org/10.2471/BLT.06.037184

Pinnock, R. \& Jones, A., 2008, 'An undergraduate paediatric curriculum based on clinical presentation and "key features", Journal of Paediatrics and Child Health 44, 661-664. https://doi.org/10.1111/j.1440-1754.2008.01377.x 
Potterton, J., Stewart, A., Cooper, P. \& Becker, P., 2010, 'The effect of a basic home stimulation programme on the development of young children infected with HIV', Developmental Medicine and Child Neurology 52, 547-551. https://doi.org/10. 1111/j.1469-8749.2009.03534.x

Ortiz, G., Koch, S., Romano, J.G., Forteza, A.M. \& Rabinstein, A.A., 2007, 'Mechanisms of ischemic stroke in HIV-infected patients', Neurology 68(16), 1257-1261. https://doi.org/10.1212/01.wnl.0000259515.45579.1e

South African Malaria, 2013, Progress and impact series report number 8 , viewed 12 September 2016, from http://www.rollbackmalaria.org/microsites/Progresslmpact September 2016, from http://ww
Series/rbm/index.php?c=16_en

South African Statistics, 2012, 'Midyear population estimates', viewed 04 June 2015, from www.statssa.gov.za/publications/SAStatistics/SAStatistics2012.pdf

Stewart, A., Wallner, P., Blecher, L., Bridgeford, T., Kirk, J. \& Salmon, M., 1994, 'Physiotherapy graduates and contribution to South Africa's health needs', South African Journal of Physiotherapy 50(3), 62-64.
UNAIDS South African, 2015, HIV and AIDS estimates, viewed 24 October 2016, from http://www.unaids.org/en/regionscountries/countries/southafrica/

Walker, S., Wachs, T., Grantham-Mcgregor, S., Black, M., Nelson, C., Huffman, S., et al., 2011, 'Inequality in early childhood: Risk and protective factors for early child development', The Lancet 378(9799), 1325-1338. https://doi.org/10.1016/ S0140-6736(11)60555-2

Wallner, P. \& Stewart, A., 1994, 'A community approach to physiotherapy', The South African Journal of Physiotherapy 50(3), 54-56.

World Confederation of Physical Therapy (WCPT), 2011, Guideline for physical therapist professional entry level education, Revised and published as a WCPT guideline at the 17th General Meeting of WCPT June, viewed 10 September 2016, from http://www.wcpt.org/sites/wcpt.org/files/files/Guideline_PTEducation complete.pdf 


\section{APPENDIX 1}

APPENDIX 1: Summary of conditions covered within the University of the Witwatersrand Physiotherapy curriculum.

\section{Neuro-musculoskeletal conditions (including paediatric cases) \\ Cardiopulmonary conditions (including}

\section{Conditions of the spine}

Muscle sprain, spasm, injury guarding

Discogenic - bulge or protrusion

Degenerative arthritis

Zygapophyseal joints (facet) - capsular strain, tear, cartilage damage,

degenerative arthritis

Fractures of the vertebral body and

spinous process

Fascia, ligament

Mechanical and inflammatory low-back pain

Inflammatory syndromes: Ankylosing spondylitis; psoriatic, reactive and rheumatoid arthritis; polymyalgia

rheumatic

Spinal hypomobility, hypermobility or instability

Hypermobility syndromes: Marfan syndrome; Ehlers-Danlos syndrome

Nerve root irritation, compression, stenosis

Spondylolysis, spondylolisthesis Spondylosis/osteoarthritis

Lumbar radiculopathy

Metabolic syndromes: osteoporosis

osteomalacia; Paget's disease

Infections: TB-spine; osteomyelitis

Neoplasms: benign or malignant

Visceral pathology: Abdominal aortic aneurysm; endometriosis; prostatitis

Fibromyalgia, myofascial pain syndrome

Piriformis syndrome, postural pain syndrome

Coccydinia, sacro-iliac joint dysfunction Thoracic outlet syndrome, T4 syndrome Visceral pain of thoracic origin Scheuermann's disease (vertebral osteochondrosis), Tietze's syndrome

Whiplash-associated disorders

Discogenic wry neck, acute locked joint

Conditions that may mimic $\mathrm{Cx}$, upper Tx or shoulder girdle symptoms: Malignant lymphadenopathy; Pancoast's tumour; Vertebral artery syndrome;

subarachnoid haemorrhage; coronary artery disease, polymyalgia rheumatic angina; carcinoma of the bronchus; diaphragmatic pleurisy; secondary malignant deposits at the scapula herpes zoster; glandular fever

Headache: cervicogenic; migraine without aura; tension type

Vertebro artery insufficiency

Temporomandibular joint dysfunction
Neurodynamics

Intraneural pathology: neuroma; demyelination; scarred epineurium arachnoiditis; irritated dura mater

Extraneural pathology: adhesion of dura to posterior longitudinal ligament; swelling of bone and muscle adjacent to a nerve trunk; narrow spinal canal

Injury as result of vascular factor; mechanical factor; axoplasmic flow, Neural fibrosis

Double crush syndrome

Neuropathy, sympathetic nervous system involvement

\section{Peripheral nerves}

Neurapraxia, axonotmesis

Neurotmesis, Erb-Duchenne paralysis or Erb's palsy (upper trunk injury)

Klumpke's paralysis (lower trunk injury)

Saturday night palsy (lower trunk injury)

Horner's syndrome, hand of

benediction, Carpal tunnel syndrome,

nerve injuries: median; radial; ulnar;

posterior tibial; common peroneal

nerve, sciatic nerve palsy, drop foot

Reflex sympathetic dystrophy - Sudeck's atrophy

Shoulder complex

Instability, Scapula dyskinesis

Subacromial impingement (primary impingement)

Secondary impingement

Supraspinatus tendonitis

Rotator cuff tear, adhesive capsulitis,

acute calcific tendonitis

Biceps tendonitis, cervical radiculitis, Pancoast's tumour

Neoplasm

\section{Orthopaedics}

Fractures and dislocation

Hand injuries, shoulder injuries

Hip and thigh injuries, elbow injuries, knee injuries

Leg injuries or pain

Foot and ankle injuries

Osteoarthritis and rheumatoid arthritis, Amputations

Fractures or dislocation of the

appendicular skeleton

Arthroplasty

Diseases of bone and joint:

Osteomyelitis, septic arthritis, avascula necrosis, gout, TB

Spinal surgery paediatric cases)

Upper respiratory infections

Complications of bed rest

Restrictive lung diseases: Pneumonia

(Pneumocystis jiroveci pneumonia

ventilator associated pneumonia other

bacterial pneumonias), TB, lung abscess,

interstitial lung diseases, acute

respiratory distress syndrome

Asthma

Bronchiectasis, atelectasis

Chronic bronchitis and emphysema

Chronic lung diseases

Burns and inhalation injuries

Diabetes mellitus, obesity, metabolic syndrome and hypertension HIV

Oncology: lung cancer, breast cancer cervical cancer

Tracheostomy management and care

Cardiac valve diseases and surgery, myocardial infarction, angina pectoris, coronary artery bypass graft surgery

Cardiac arrhythmias and cardiac failure

Chest trauma: rib fractures, sternal

fractures, pneumothorax, haemothorax,

pleural effusions, empyema

Lower respiratory tract surgery

Pre- and post-operative management of surgical patients

Respiratory failure, shock states

Acute renal failure

Acute brain injuries

Acute spinal cord injuries

Polytrauma patient in ICU

Guillain-Barre patient in ICU

Cystic fibrosis

Sputum induction

ICU, intensive care unit; TB, tuberculosis.

Neurological conditions (including paediatric cases)

Stroke

Traumatic brain injury

Spinal cord injury

Parkinson's disease

Motor neuron disease

Multiple sclerosis

Guillain-Barre syndrome

Prematurity

Cerebral palsy: ataxia, hypertonicity,

hypotonicity, athetosis

Spina bifida

Spinal muscular atrophy

Duchenne muscular dystrophy

Developmental coordination disorder HIV

Pneumonia

Cystic fibrosis

Bronchiectasis

Congenital cardiac lesions

Developmental delay because of a number of factors

Haemophilia

Head injury

Meningitis

Burns

Hydrocephalus

Fractures

Scoliosis

Post-operative complicated selective

orthopaedic surgery, for example,

single-event multilevel surgery (SEMLS)

Sports injuries in kids

Myasthenia gravis

Epilepsy, neurosurgery

Autism

Normal development

Common neonatal disorders

Non-specified categories

Geriatrics

Women's health

Occupational health

Metabolic disorders

Tumours and disorders of growth

Chronic kidney disease

Chronic liver disease and hepatic failure

Parathyroid disorders

Systemic lupus erythematosus

General urology

Primary skin diseases 